\title{
A Novel Adaptive Rood Pattern Search Algorithm
}

\author{
Vanshree Verma, Sr. Asst. Prof. Ravi Mishra \\ Department of Electrical and Electronics Engineering, Shri Shankaracharya College of Engineering \& \\ Technology. Junwani, Bhilai (C.G.)
}

\begin{abstract}
This paper is based upon Adaptive Rood Pattern Search algorithm for Block Matching Algorithm. There are various types of algorithm is available but this method is most reliable. Experimental results shows that it gives better results than other algorithms. This algorithm uses motion activity of the video. In this paper, adaptive rood pattern search method using motion activity is applied on videos.
\end{abstract}

Keywords: motion activity, segmentation, motion vectors, PSNR.

\section{Introduction}

Several advantages have been made in digital technology recently in order to make videos more valuable and useful for users. Therefore, the development of methods for indexing and retrieving videos is necessary. Video shot boundary detection is the fundamental step of indexing, browsing and retrieval applications. Shot boundary detection is the most basic video segmentation task. General objective is to segment a given video sequence into is constituent shots and to identify and classify the different transitions between adjacent shots in the sequence. The first step of any video processing method is to segment the input video into elementary shots. A shot is defined as the continuous frame from start to the end of recording in the camera. It shows continuous image in sequence. There are two types of transitions that can occur between shots, they are abrupt change and gradual transition. Shot detection identify and classify this different shot transitions in the sequence.

Various algorithms have been proposed but commonly used method is Block Matching Algorithm (BMA). It is a commonly known motion estimation technique used to measure the motion of moving objects. Several BMAs have been proposed in recent years. In this paper, Adaptive Rood Pattern Search algorithm (ARPS) is used to detect shot transitions. Section II gives an introduction to BMA, section III focused on methodology of ARPS, section IV gives some experimental results and section V is conclusion and section VI is references.

\section{Block Matching Algorithm}

To detect shot in a given video sequence, first estimation of the motion of the moving object is performed. The basic idea of method of segmentation is that images in the neighbour of a transition are relatively highly dissimilar. It then measures the discontinuities in the video stream. It first detect the motion vectors between image $i$ and image $i+2$ then determine the intensity of motion, repeat this process until reaching the last frame of the video and compares the difference between the intensities of successive motion to a specified threshold. The idea can be visualized in fig.1.

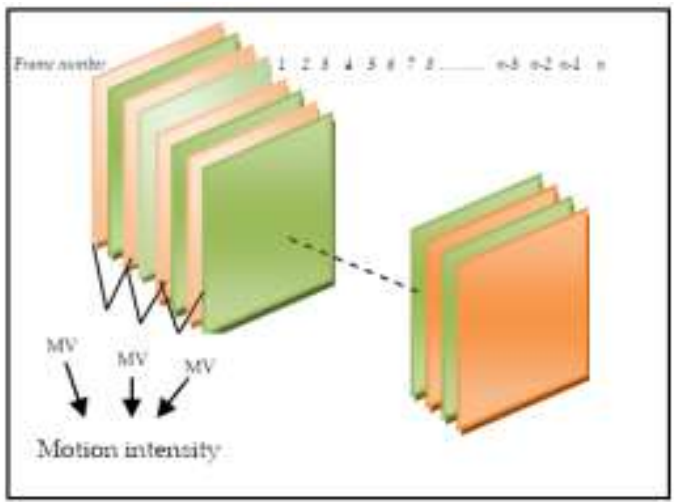

Fig.1 The idea of video segmentation using motion intensity

Commonly known motion estimation technique is Block matching algorithm. In this algorithm, first a frame is divided into blocks of $\mathrm{M} \times \mathrm{N}$ pixels or square locks of $\mathrm{N} \times \mathrm{N}$ pixels. Then, each block undergoes translation only 
with no scaling or rotation. The blocks in the first frame are compared to the blocks in the second frame. Then, for each current block, we find that best match for the current block in the neighborhood to calculate motion vectors. Motion Vectors can be calculated for each block by observing where each block from the first frame ends up in the second frame.

Block matching divides the current frame into macro blocks that are then compared with reference block and its adjacent neighbors in the previous frame to create a vector that defines the motion in a macro block from one frame to another frame. This movement calculated for all the macro blocks constitutes the motion estimation of the current frame. To find the motion vectors for a current block, a search parameter $p$ is used. The neighborhood of current block match is constrained up to p pixels on all fours sides of the macro block in the frame. For large motion activity, a larger $\mathrm{p}$ is required, and hence the computational process of motion vectors becomes more expensive. The macro block, for example, is taken as a square of side 16 pixels, and the search parameter $\mathrm{p}$ is 7 pixels. The matching of one macro block with another is based on the output of a cost function. There are various cost functions, of which the most popular and less computationally expensive is Mean Absolute Difference (MAD) given by equation (i). Another cost function is Mean Squared Error (MSE) given by equation (ii).

$$
\begin{aligned}
& M A D=\frac{1}{N^{2}} \sum_{i=1}^{K-1 N-1} \sum\left|C_{j}-R_{j}\right| \\
& M S E=\frac{1}{N^{2}} \sum_{i=1}^{N-1} \sum_{j=0}^{N-1}\left(C_{j}-R_{j}\right)^{2}
\end{aligned}
$$

Where $\mathrm{N}$ is the side of the macro bock, Cij and Rij are the pixels being compared in current macro block and reference macro block, respectively. Peak-Signal-to-Noise-Ratio (PSNR) given by equation (iii) characterizes the motion compensated image that is created by using motion vectors and macro clocks from the reference frame.

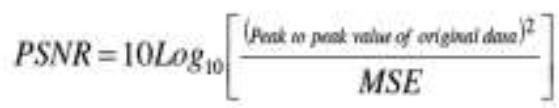

Various BMAs have been developed in recent years for motion estimation in videos. They are Exhaustive Search, Three Step Search, New Three Step Search, Simple and Efficient Search, Four Step Search, Diamond Search, Adaptive Rood Pattern Search method. Among all these method, ARPS is the best and effective matching algorithm. Next section gives an introduction and steps of ARPS method.

\section{Adaptive Rood Pattern Search Method}

This algorithm uses the motion vector of the current block to detect the motion activity. Motion activity is one of the motion features that used to describes the intensity of activity or motion. The magnitude of motion vector represents the measure of intensity of moving object. To extract motion intensity, video sequence is transformed into gray level frames. Then each frame is divided into macroblocks of $16 \times 16$ size. Then motion vector is calculated by determining the intensity of motion in frame. ARPS uses this motion vector to detect shot.

In ARPS algorithm, we first assume that in general the motion in a frame is coherent. If the macroblocks around a current macroblock are moving in a particular direction then there is a high possibility that the current macroblock will also move in that direction. This method uses the motion vector of macroblock to compare it with its immediate left block to detect its motion vector. An example is shown in fig 9. Here step size of $\mathrm{S}=\operatorname{Max}(|\mathrm{X}|,|\mathrm{Y}|)$ is used where $\mathrm{X}$ and $\mathrm{Y}$ are the $\mathrm{X}$-coordinate and y-coordinate of the predicted motion vector. This rood pattern search is always the first step. It directly puts the search in an area where there is a high probability of finding a good matching block.

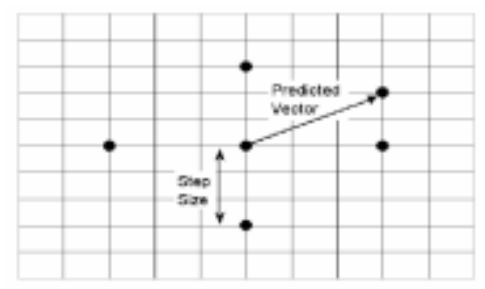

Fig 2. Adaptive Root Pattern Search Method 
In order to obtain an accurate motion vector prediction of the current block, two factors need to be considered: 1) choice of the region of support that consists of the neighboring blocks whose motion vectors will be used to calculate the predicted motion vector, and

2) Algorithm used for computing the predicted motion vector.

The steps of ARPS algorithm is described below:-

Step 1: Compute the matching error using mean absolute difference (MAD) between the current block and the block at the same location in the reference frame i.e., the center of the current search window.

If MAD is less than threshold then motion vector is zero otherwise

Pattern size=Max $\{\mid \mathrm{MV}$ predicted(x), MV predicted(y) $\mid\}$

Go to Step 2.

Step 2: Align the center with the center point of the search window and check its four search points plus the position of the predicted motion vector to find out the current MSE point.

Step 3: Set the center point at the MSE point found in the previous step and check points. If the new MSE is not at the center of the current point, repeat this step; otherwise, the motion vector is found.

\section{Experimental Results}

The main aim of shot detection is to detect the transitions in video sequence and make it useful for users. It remove the redundancy that present in videos and make the video more exciting and interesting. Here are some experimental results of videos. We calculate the number of shots, number of shots correctly reported, number of false detections. Also we assume a threshold value to determine motion vector. An example is shown in figure 3 and shot detection is 40 .

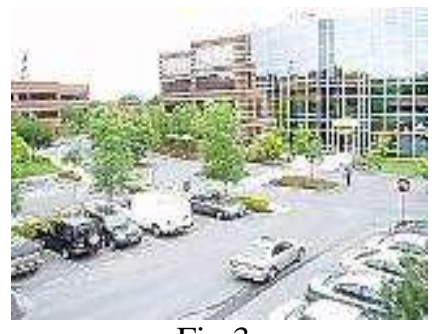

Fig 3.

Fig 4 gives an example of shot boundary detection in a news video sequence. First frame of each frame is considered as the key image of the detected shot.

Fig 4
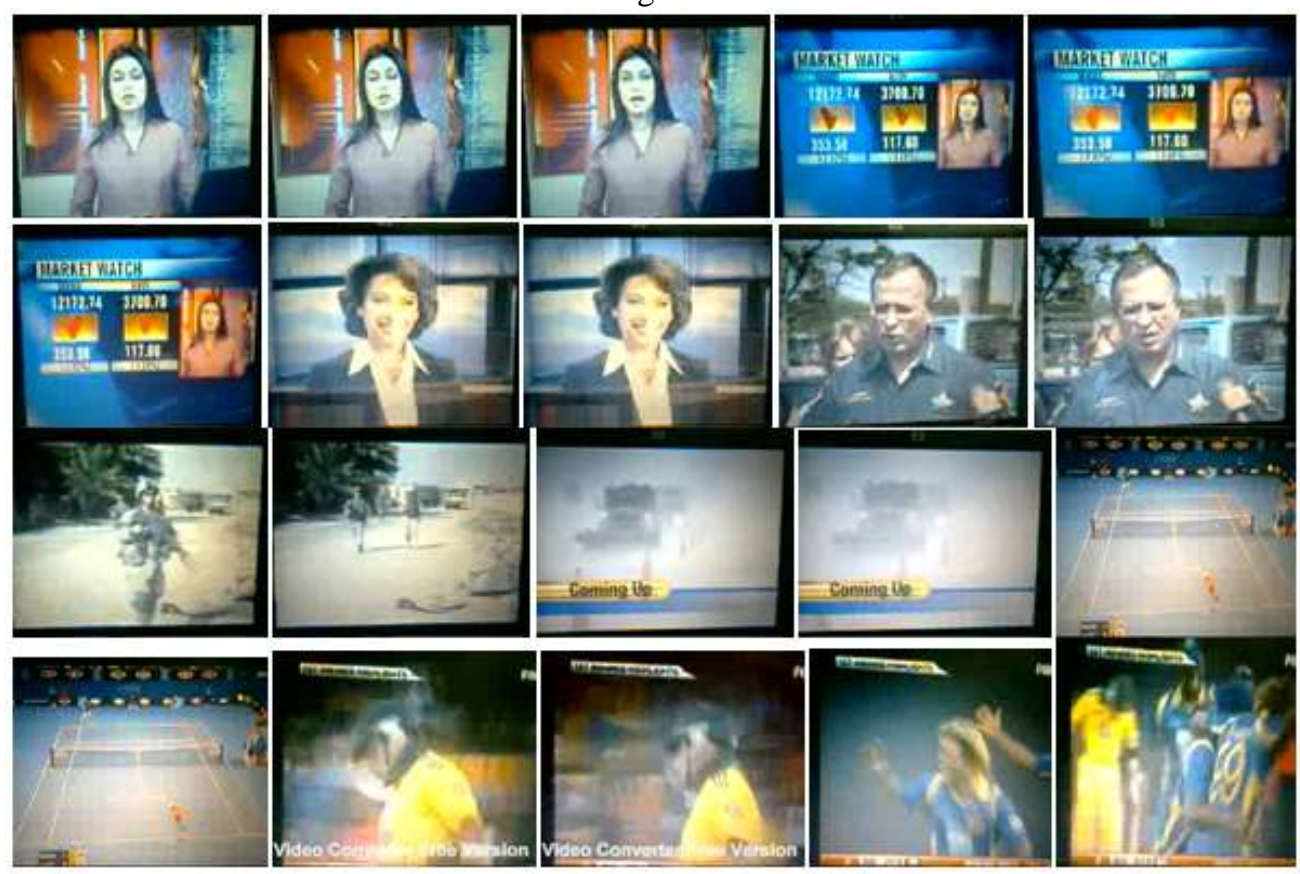

www.iosrjournals.org 
Another example of the of the motion vector description technique using matlab is given by fig 5 .

(a)

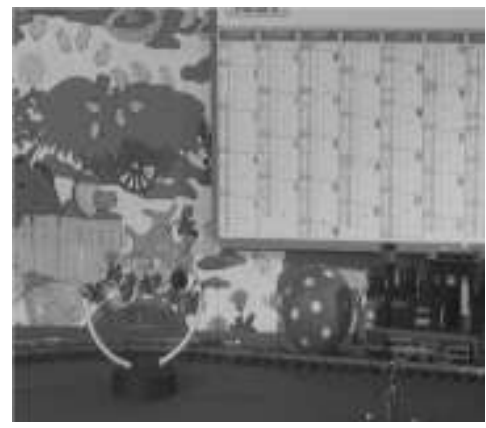

(b)

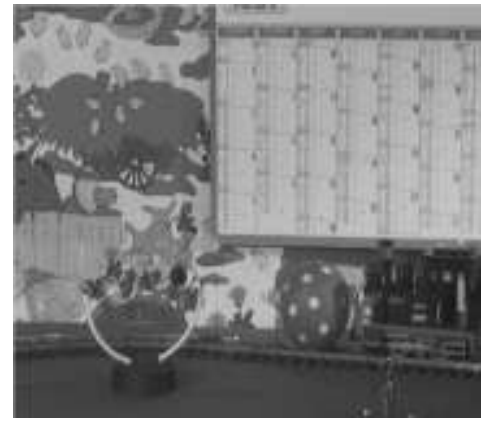

(c)

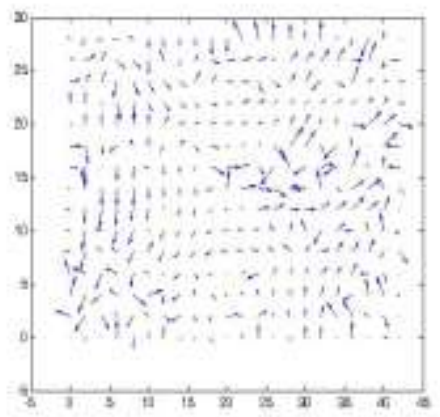

Fig 5. (a) and (b) two successive caltrain frames and (c) motion vector result of (a) and (b) frames.

Search points per macroblock and Peak Signal to Noise Ratio (PSNR) of Block matching algorithms is shown in fig 6 and 7 .

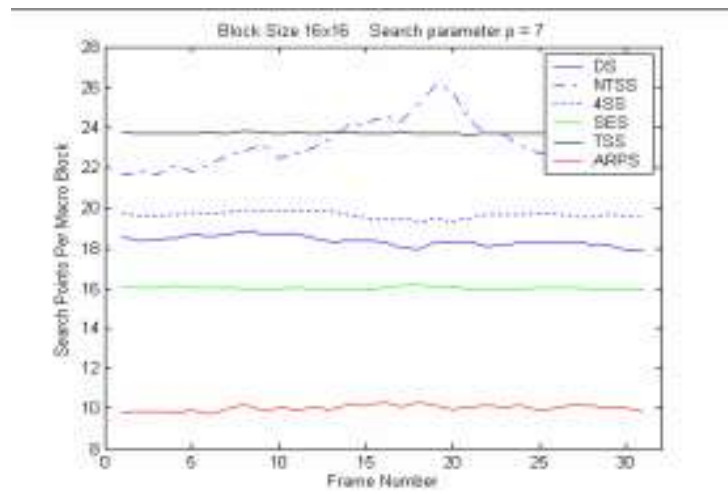

Fig.6. Search points per macro block while computing the PSNR performance of Fast Block Matching Algorithms.

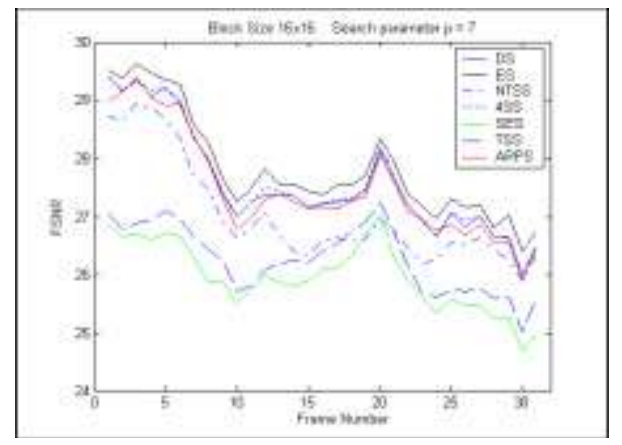

Fig.7. Peak-Signal-to-Noise-Ratio (PSNR) performance of Fast Block Matching Algorithms.

Caltrain Sequence was used with a frame distance of 2. 


\section{Conclusion}

This paper gives ARPS method in detail. We have applied block matching algorithm for various videos and we got that ARPS gives better and more reliable results than other algorithms and the use of motion activity increases the rate of shot detection. This algorithm can also give better performance and result in real time implementation.

\section{References}

[1] Video shot boundary detection using motion activity descriptor Abdelati Malek Amel, Ben Abdelali Abdessalem and Mtibaa Abdellatif, april 2010

[2] Aroh Barjatya, "Block Matching Algorithms For Motion Estimation, "Student Member, IEEE, DIP 6620 Spring 2004 Final Project paper.

[3] Dr.V.K.Ananthashayana and Pushpa.M.K, "Joint Adaptive Block Matching Search (JABMS) Algorithm for Motion Estimation", International Journal of Recent Trends in Engineering, Vol 2, No. 2, November 2009

[4] Yao Nie, and Kai-Kuang Ma, "Adaptive Rood Pattern Search for Fast Block-Matching Motion Estimation", IEEE Trans. Image Processing, vol 11, no. 12, pp. 1442-1448, December 2002.

[5] D. A. X. Sun and B. S. Manjunath, "A Motion Activity Descriptor and Its Extraction in Compressed Domain," IEEE Pacific-Rim Conference on Multimedia (PCM), vol. LNCS 2195, pp. 450-453, October 2001.

[6] Don Adjeroh, M. C. Lee, N. Banda, and Uma Kandaswamy, "Adaptive Edge-Oriented Shot Boundary Detection," Hindawi Publishing Corporation EURASIP Journal on Image and Video Processing Volume 2009, Article ID 859371, 13 pages doi:10.1155/2009/859371, 18 May 2009.

[7] Amel Malek Abdelati, Jlassi bahaeddine, Abdessalem Ben Abdellali and Abdellatif Mtibaa, " Suivi d'objet en movement par la method Level Set variationnelle, " international workshop on system engineering design \& application, October 24-26, 2008, Monastir, Tunisia.

[8] S. Jeannin and A. Divakaran, "MPEG-7 visual motion descriptors, "IEEE Transactions on Circuits and Systems for Video Technology, vol. 11, pp. 720-724, 2001 